\title{
Stressed backbone and elasticity of random central-force systems
}

\author{
C. Moukarzel * \\ Höchstleistungsrechenzentrum, Forschungszentrum Jülich, \\ D-52425 Jülich, Germany. \\ P. M. Duxbury \\ Dept. of Physics/Ast. and Center for Fundamental Materials Research, \\ Michigan State University, East Lansing, MI 48824, USA.
}

We use a new algorithm to find the stress-carrying backbone of "generic" site-diluted triangular lattices of up to $10^{6}$ sites. Generic lattices can be made by randomly displacing the sites of a regular lattice (see Fig. 1). The percolation threshold is $p_{c}=0.6975 \pm 0.0003$, the correlation length exponent $\nu=1.16 \pm 0.03$ and the fractal dimension of the backbone $D_{b}=1.78 \pm 0.02$. The number of "critical bonds" (if you remove them rigidity is lost) on the backbone scales as $L^{x}$, with $x=0.85 \pm 0.05$. The Young's modulus is also calculated.

*Present Address: Instituto de Fisica, Universidade Federal Fluminense, Niteroi RJ, Brazil. e-mail: cristian@if.uff.br 
The forces between atoms can often be divided into two classes "central forces" and "angular forces" (e.g. covalent bonds). In engineering, structures composed of bars connected at nodes (e.g. some bridges), get their rigidity primarily from the tensile and compressive stiffness of the bars (these are central-force terms). Structures of this sort are called "trusses", while those in which the angle forces (or beam-bending) are important are called "frames". It is simple to see that systems which are dominated by angle forces support an applied stress as long as they are simply connected. In contrast, systems with only central forces require higher order connectivity, the simplest rigid structure being a triangle. In many applications; for example in granular media ${ }^{1}$, glasses $^{2}$, gels ${ }^{3,4}$ and in engineering design, the disorder in a central-force structure is important and must be considered. The stress-bearing paths of central-force systems have been primarily studied by brute-force solution of the force equations ${ }^{5-8}$. Although useful and important, this method is slow and subject to roundoff errors for large structures. An efficient method for relating the connectivity of a central-force structure to its ability to carry stress is an important and, in general unsolved, problem. One exception to this is two-dimensional random lattices, for which exact conditions ${ }^{9-12}$ relating connectivity to "rigidity" have existed for over a decade. However, till recently ${ }^{13}$ there has been no efficient implementation of these conditions, and their associated algorithms in either physics or engineering. This paper and the preceding paper by Jacobs and Thorpe $(\mathrm{JT})^{13}$ describe the first implementations of these ideas. We use our algorithm, to calculate the stressed backbone, and in combination with an iterative solver, to find the elastic properties of these backbones. We also identify the critical (red) bonds as those whose removal would lead to loss of rigidity, and study their scaling properties. For reasons outlined below these methods apply to randomly displaced (or "generic" - see Fig. 1a) central-force lattices. a)

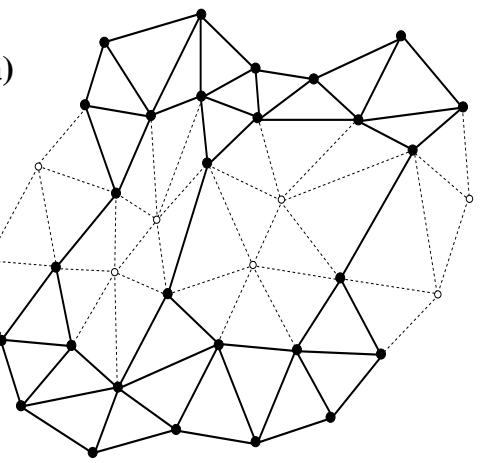

b)

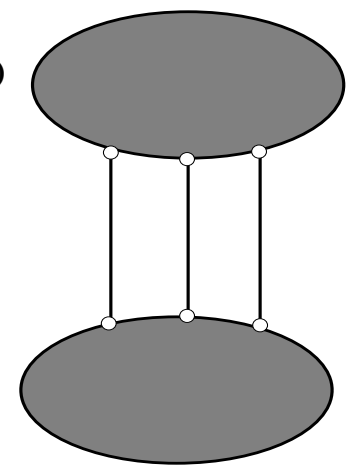

FIG. 1. A configuration that is unstable to shear on a regular lattice, but is stable on a displaced lattice (dotted lines indicate absent bonds). a) The configuration in the "bar-joint" representation (28 joints and 53 bars). b) The configuration in the "body-bar" representation (2 bodies and 3 bars).

Our ability to determine, from connectivity information alone, whether a central-force structure contains a stress carrying path is based on Laman's theorem ${ }^{9}$.

Laman's theorem $A$ random lattice (see below for a precise definition) consisting of $N$ nodes and $B$ bonds so that $2 N-B=3$ is rigid if and only if there is no subset of the lattice, consisting of $n$ nodes connected by $b$ bonds, for which $2 n-b \leq 3$ is violated.

This is the "bar-joint" statement of Laman's theorem. The origin of the expression $2 n-b=3$ is easy to understand. Each node (joint) in two dimensions has two degrees of freedom (two translations), and each bond (bar) is a constraint (for example in Fig. 1a, $n=28, b=53$ ). In the expression $2 n-b=3$, the 3 is there because in two dimensions a rigid body (in this case the whole lattice or cluster) has 3 degrees of freedom (two translations and a rotation). $2 n-b=3$ is the two dimensional version of a general constraint counting argument introduced by Maxwell.

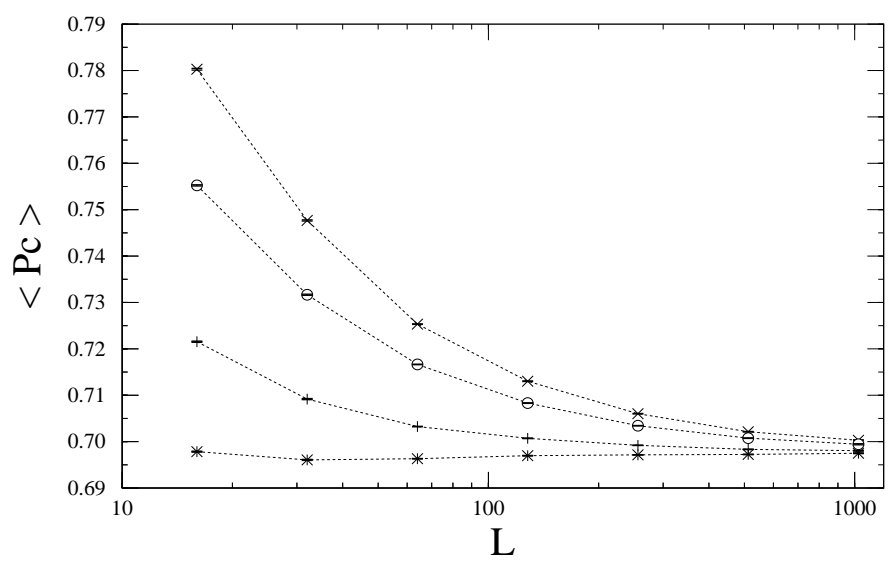

FIG. 2. The rigidity threshold as a function of sample size. AS with periodic boundary conditions $(*)$, AS with open boundary conditions $(+)$, IS with open boundary conditions $(\times)$ IS with periodic boundary conditions $(\circ)$. The lattice sizes (L) (number of configurations) used are as follows; $16\left(2 \times 10^{5}\right)$, $32\left(10^{5}\right), 64\left(8 \times 10^{4}\right), 128\left(2 \times 10^{4}\right), 256\left(1.2 \times 10^{4}\right), 512\left(2 \times 10^{3}\right)$, $1024\left(2 \times 10^{2}\right)$.

However the new feature here is that constraint counting is exact in two dimensions provided it is implemented at all length scales (Unfortunately this result does not extend to three dimsensions, where counterexamples ${ }^{12}$ to the three-dimensional extension of this argument, $3 n-b=6$, are known to exist). However, even in 2$\mathrm{d}$ a naive algorithm must check all subclusters of a set of $N$ nodes and so is not polynomial complete. However Laman's theorem may be implemented by using the "bipartite matching" algorithm from graph theory ${ }^{12}$, which, when refined as described below, scales as $N^{1.15}$ for finding the stressed backbone at the rigidity percolation point.

Our implementation of Laman's theorem is a cluster labelling algorithm ${ }^{21}$. Although we do site percolation, where $p$ is the probability that a site is occupied, the algorithm works by testing a newly added bond against the configuration of rigid clusters already on the lattice. For a given $p$, we find the site configuration, and from it the configuration of present bonds. Then we start with an empty lattice and add the present bonds one at a time. Each rigid cluster is a "body" with 3 degrees of freedom, so we must generalise the statement $2 n-b=3$ of the original lattice to $3 n_{\text {bod }}-b=3$, where $n_{\text {bod }}$ is the num- 
ber of bodies (or rigid clusters) in a configuration. For example the configuration of Fig. 1a, has two bodies and 3 bars (see Fig. 1b). A key component of the algorithm is the realisation by Hendrickson ${ }^{12}$ that it is easy to determine whether a bar (bond) is redundant with respect to the bonds that are already in the lattice.

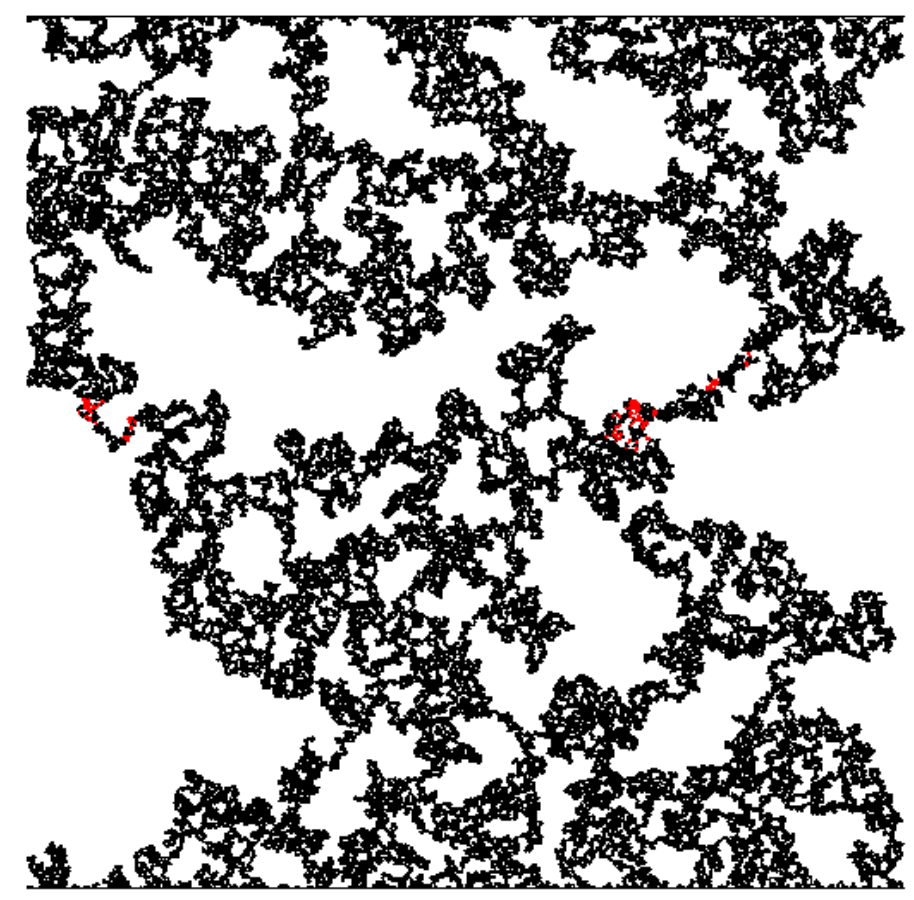

FIG. 3. The stressed (AS) backbone on a lattice of size $L=1024$ with open boundaries.

If the bonds of the lattice are replaced by Hooke's springs, a redundant bond leads to internal stresses in the spring lattice. A redundant bond causes a violation of the condition $3 n_{b o d}-b=3$ by adding an extra spring to the lattice. The algorithm checks to see if $3 n_{b o d}-b=3$ is satisfied by using "bipartite matching" 12 or "the pebble game" 12,13 to see if each of the bodies' degrees of freedom can be "matched" to the bonds of the configuration (note that JT use the "pebble game" in the original bar-joint representation).

If an extra or redundant spring is added to a cluster that is already rigid (i.e. already satisfies $3 n_{\text {bod }}-b=3$ ), the matching algorithm identifies the bonds which become internally stressed when the extra spring is added. We then give these internally stressed bonds the same cluster label. In this way we find "internally stressed (IS) clusters". Finally, an applied tensile stress can be mimiced by adding two rigid beams to the two opposite sides of the lattice, and then by adding a fictitious bond between the rigid beams. In this way, we determine when the lattice can support an applied tensile stress. Full details of the algorithm will appear elsewhere ${ }^{21}$. In JT, the bond probability is fixed. Our algorithm is complimentary to theirs as we add bonds one at a time, so we find the percolation concentration exactly for each sample. We chose this method not only because it is very efficient (comparable to JT), but also so that we can find the exact backbone for each sample, and hence the "critical (or red) bonds" of the backbone (see below).
There are several ways to define the onset of stress transmission through a lattice. The two which are most physically appealing are:

1. The point at which an applied stress $(A S)$ is transmitted across the lattice and;

2. The point at which internally stressed regions (IS) connect together to form stressed clusters of macroscopic size.

Both of these definitions have simple representations in terms of a lattice of Hooke's springs. The first (AS) corresponds to a random Hooke's spring lattice to which, for example, a tensile stress is applied, while the second (IS) corresponds to the internal stresses in a random Hooke's spring lattice with random natural lengths. We study the stressed backbone of these lattices as a function of site dilution. We also tested the effect of boundary conditions on these two definitions of rigidity percolation, because a local change in rigidity (e.g. by adding a bond) can be transmitted over long distances so boundaries might be more important in this problem than in connectivity percolation. However, we find that in the large-lattice limit both the AS and IS percolation definitions lead to the same, boundary condition independent, threshold. This behavior is presented in Fig. 2, from which we find that $p_{c}=0.6975 \pm 0.0003$. On regular lattices, previous work ${ }^{15}$ using direct solution of the force equations lead to estimates close to $p_{c}=0.713$ for samples of up to size $L=75$. As can be seen from Fig. 2, this is consistent with the result on random lattices, although at that lattice size, there is still considerable dependence on boundary conditions. However, in general there is no reason to believe that the percolation threshold on random lattices should be the same as that on regular lattices. This difference is illustrated by the configuration of Fig. 1b. On a regular lattice that configuration is not rigid to shear, but if the lattice sites are displaced, it becomes rigid. That is because on a regular lattice, the three bars are parallel, so these constraints are "degenerate". Thus for that configuration, the random lattice is more rigid than the regular lattice. 


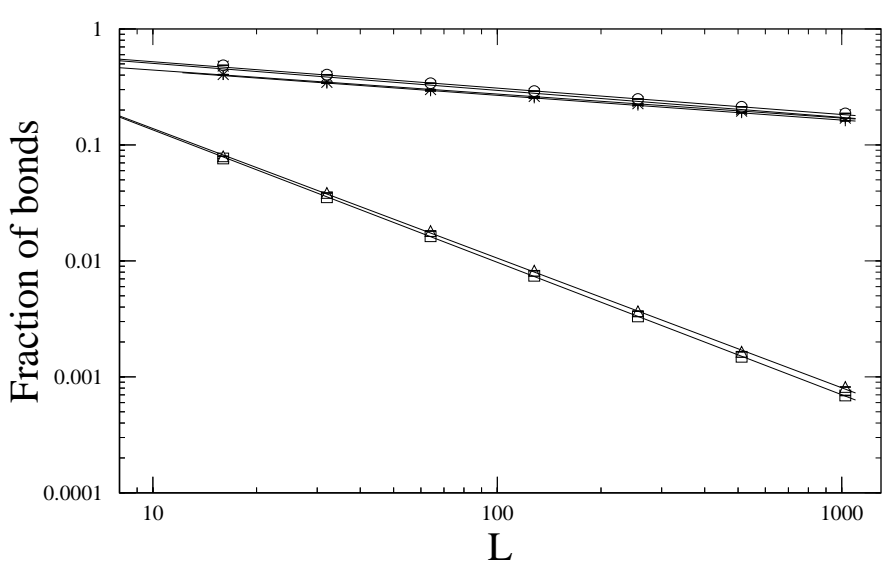

FIG. 4. a) Finite-size-scaling plot of the normalised number of bonds $N_{B} / L^{2}$ on the stressed backbone at the percolation point for: AS with periodic b.c.'s $\left(^{*}\right)$; AS with open b.c.'s $(+)$; IS with periodic b.c.'s (X) and ; IS with open b.c.'s $(\mathrm{O})$. b) Finite-size-scaling plot of the normalised number of "red" bonds on the backbone, $N_{R} / L^{2}$ (AS with periodic b.c's). The lattice sizes used and number of configurations were the same as for Fig. 2 .

However it is easy to construct configurations which are more rigid on a regular lattice (e.g. a sequence of aligned bonds forming a "guy" wire), so it is unclear as to whether displaced lattices have a lower or higher $p_{c}$ than regular lattices.

From the variation in the percolation concentration $\Delta p_{c} \sim L^{-1 / \nu}$, we are able to find the correlation length exponent. We did this for three ways of defining $\Delta p_{c}$, namely:

a) $\left(p_{c}(L)-p_{c}(\infty)\right)$;

b) $\left(p_{c}^{\text {open }}-p_{c}^{\text {periodic }}\right)$ and;

c) $\sqrt{ }\left(<p_{c}^{2}>-<p_{c}>^{2}\right)$,

and for several types of boundary conditions in each case. From these extensive calculations, we find $\nu=1.16 \pm 0.03$. Although it is not the main focus of this paper, we note that in $\mathrm{JT}$, it is claimed that the infinite cluster, $P_{\infty}$ (which includes internally stressed bonds (stressed backbone), and unstressed bonds which satisfy $2 n-b=3$ ) has a fractal dimension around $D_{f} \sim 1.86$.

If we assume a second order behavior in $P_{\infty}$, we find a similar fractal dimension. However, a mean field theory ${ }^{4}$ suggests that the rigidity transition is first order (so $D_{f}=2$ in 2-d), and similarly on Bethe lattices the rigidity transition is first order ${ }^{22}$. Thus we have also tested the possibility of a first order transition ${ }^{23}$ in $P_{\infty}$, and find that the data is consistent with a weakly first-order transition, with the first-order jump $\Delta P_{\infty} \sim 0.085$ at $p_{c}$. However, even larger lattices (up to of order $L=10,000$ ) are needed to determine convincingly whether, in $2-\mathrm{d}, P_{\infty}$ is first order.

An example of a stressed backbone at the percolation point is presented in Fig. 3. We measured the number of bonds on backbones such as that shown in Fig. 3, and the results of a scaling plot are presented in Fig. 4. From this figure, we find $D_{b}=1.78 \pm 0.02$. This backbone dimension is different than that for connectivity percolation where the backbone dimension is $1.62 \pm 0.01$, and it is also considerably larger than that of the stressed back- bone of regular triangular lattices $1.64 \pm 0.05$ found by direct solution ${ }^{8}$ on small lattices (up to $L=80$ ). The latter discrepancy could be due to a fundamental difference between the random and regular lattices, but it also could be due to imprecise estimates of the percolation concentration in previous work due to finite size effects (see Fig. 2). At the percolation point, there are a set of bonds whose removal leads to loss of backbone rigidity. We calculated the number of these critical red bonds, $N_{R}$, and their scaling behavior at the percolation point is also shown in Fig. 4. They scale as $N_{R} \sim L^{x}$, with $x=0.85 \pm 0.05$. This is consistent with $x=1 / \nu$, although we have no analytic argument for why this should be so. Previous work on the elastic exponents of regular triangular, central force, networks have produced conflicting results. Although the early work ${ }^{5}$ gave an exponent in the range, $1.3 \leq f / \nu \leq 2.0$, later work suggested that the central force and bond-bending (angular force) problems were in the same universality class $^{8,15,16}$, so that ${ }^{17}$ $f / \nu \sim 3.0$. There have even been suggestions ${ }^{15,16}$ that in 2 -d, site percolation has exponent near $f / \nu \sim 1.0$, while bond percolation has exponent near $f / \nu \sim 3.0$. Finally, there is a recent mean field theor ${ }^{4}$ which gives exponent $f \sim 1.5$.

The difficulty in obtaining good estimates have been ascribed to: a) unusually strong accumulation of roundoff errors $^{7}$, and b) lack of precision ${ }^{8}$ in the estimate of $p_{c}$. We find that roundoff errors are largely eliminated if we use the graph theory method to remove all non-stressed bonds before applying the conjugate gradient method. In addition we know $p_{c}$ exactly for each configuration, so we do not have to study a range of $p$ using an interative solver. Thus we have been able to study the elastic constants for lattice sizes which were previously inaccessible (up to linear size $L=512$ - see Fig. 5).

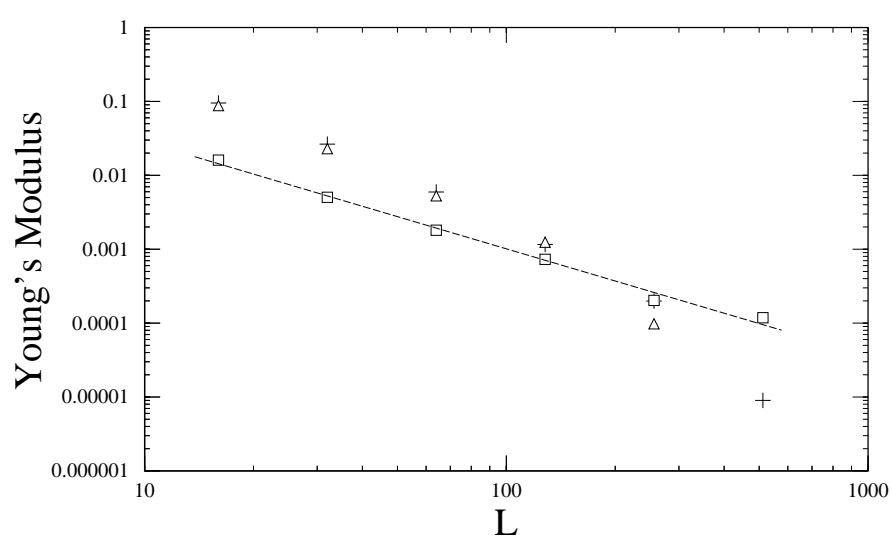

FIG. 5. Finite-size-scaling behavior of the Young's modulus for: regular lattices $(+)$; lattices randomly displaced by $0.2(\triangle)$ and; lattices with random bond angles $(\square)$. The lattice sizes used (number of configurations) were as follows; 16(20,000), 32(10,000), 64(1,000 - 2,000), 128(100-300), $256(20-40), 512(4-6)$.

As expected, a certain number of the "generic" backbones are not rigid on a regular lattice due to degeneracies. However, the fraction of the backbones that are nonrigid on regular lattices increases very slowly with lattice size, and is still only $\sim 50 \%$ at $L=512$. Now note that 
if the sites of a generic backbone, which is non-rigid on a regular lattice, are displaced by a small amount $\Delta$, the elastic modulus of that backbone is $O\left(\Delta^{2}\right)$. Thus, the elastic constants of generic backbones are usually nonuniversal, for sizes accessable to simulation, even for lattices displaced by 0.2 (see Fig. 5).

To avoid the slow size effect caused by proximity to the regular lattice limit, we also studied a model where the locations of the elements of the elastic matrix were set by the connectivity of the backbone. To mimic the highly displaced lattice (large $\Delta$ ) limit, we assign each bond an angle to the $\mathrm{x}$-axis which is drawn from a random distribution of angles (on the interval [0,360], and calculate the elastic constant using these angles in the force equations. The results for this "random angle model" are also shown in Fig. 5 (each present bond has unit spring constant). We found that the value $f / \nu \sim 1.45$ is quite universal in this limit.

Acknowledgements We acknowledge support from PRF, by the DOE under contract DE-FG02-90ER45418, and by the Humboldt foundation (PD). We thank Paul Leath, Bruce Hendrickson, Mike Thorpe and Don Jacobs for useful discussions.

\section{References}

${ }^{1}$ E. Guyon, S. Roux, A. Hansen, D. Bideau, J.-P. Troadec and H. Crapo, Rep. Prog. Phys. 53, 373 (1990)

2 J.C. Phillips, J. Non-Cryst. Sol. 43, 37 (1981); M.F. Thorpe, J. Non-Cryst. Sol. 57, 355 (1983)

3 M. Rubinstein, L. Leibler and J. Bastide, Phys. Rev. Lett. 68, 405 (1992)

4 S.P. Obukhov, Phys. Rev. Lett. 74, 4472 (1995)

5 S. Feng and P.N. Sen, Phys. Rev. Lett. 52, 216 (1984)

6 M.A. Lemieux, P. Breton and A.-M.S. Tremblay, J. de Physique 46, L-1 (1985)

7 A.R. Day, R.R. Tremblay and A.-M.S. Tremblay, Phys. Rev. Lett. 56, 2501 (1986)

8 A. Hansen and S. Roux, Phys. Rev. B40, 749 (1989)

9 G. Laman, J. Eng. Math. 4, 331 (1970)

${ }^{10}$ L. Lovasz and Y. Yemini, Siam J. Alg. Disc. Meth. 3, 91 (1982)

11 A. Recski, Disc. Math. 108, 183 (1992)

12 B. Hendrickson, Siam J. Comput. 21, 65 (1992); Bruce Hendrickson, private communication.

13 D. Jacobs and M.F. Thorpe to be published

14 M.F. Thorpe and E.J. Garboczi, Phys. Rev. B35, 8579 (1987)

15 S. Arbabi and M. Sahimi, Phys. Rev. B47, 695 (1993)

16 M. Knackstedt and M. Sahimi, J. Stat. Phys. 69, 887 (1992)

17 J.G. Zabolitzky, D.J. Bergman and D. Stauffer, J. Stat. Phys. 44, 211 (1986)

20 See for example: C. H. Papadimitriou and K. Steiglitz, "Combinatorial Optimization: Algorithms and Complexity", Prentice Hall, 1982.

21 C. Moukarzel, J. Phs. A: Math. Gen. 29 (1996), 8097; ( physics/9612013).

22 C. Moukarzel, P.M. Duxbury and P.L. Leath, to be published.

${ }^{23}$ C. Moukarzel and P.M. Duxbury to be published. 